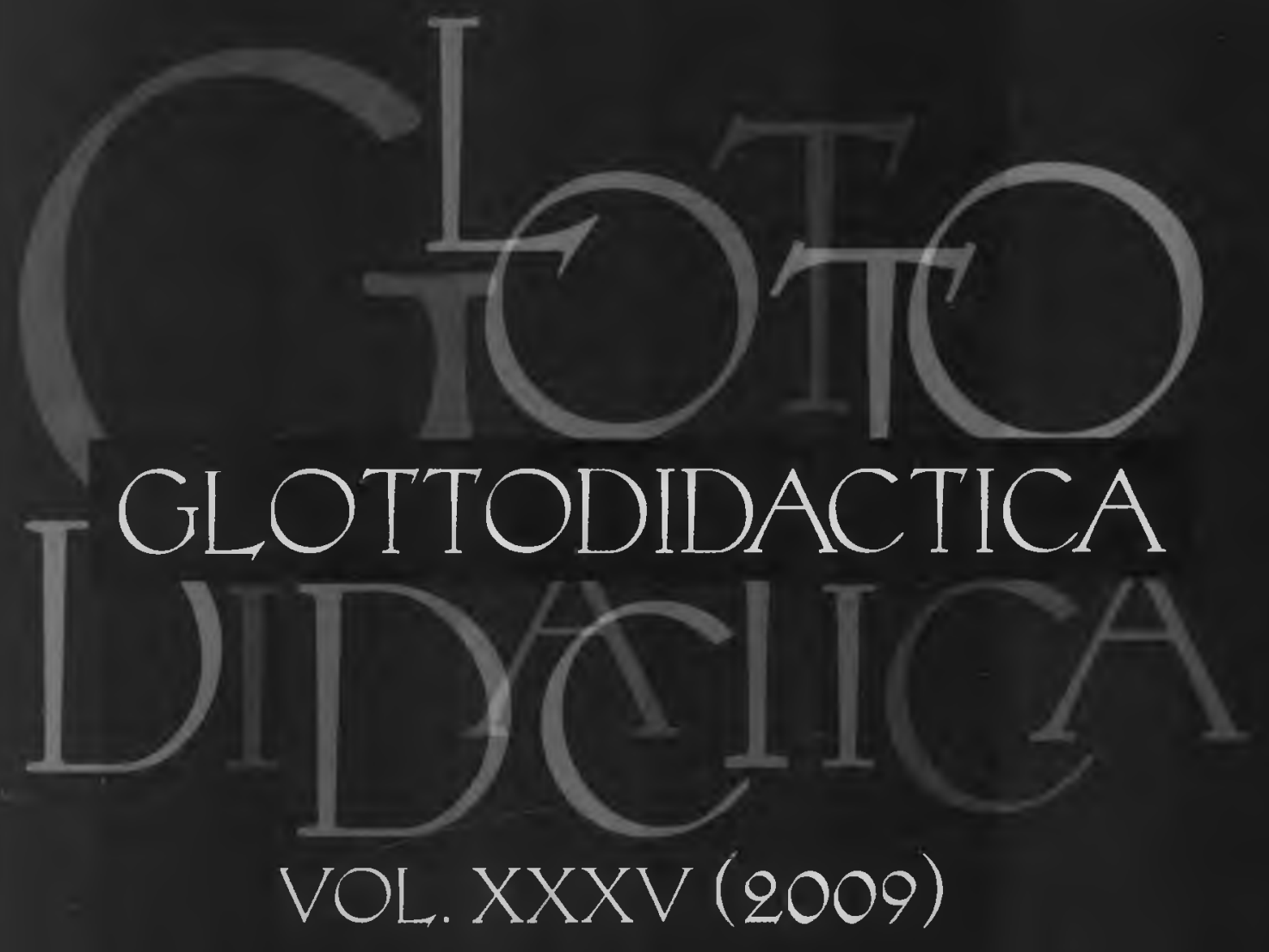

WYDAWNICTWO NAUKOWE UAM 



$$
\operatorname{cod} .42582 \wedge \frac{11}{1309}
$$

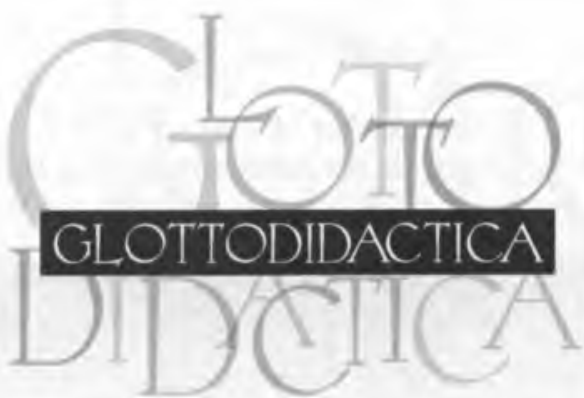




\section{GLOTTODIDACTICA \\ An International Journal of Applied Linguistics \\ Founding Editor: Prof. Dr. Ludwik Zabrocki}

Ausgabe / Issue 35

Herausgeber / Publisher

Institut für Angewandte Linguistik der Adam Mickiewicz Universität Poznań

V.i.S.d.P. Responsible person in accordance with Polish press law

Prof. Dr. Waldemar Pfeiffer (Honorary Editorship)

Redaktion (Leitung) / Editorial Head

Prof. Dr. Barbara Skowronek

Redaktionelle Mitarbeit / Editorial support

Dr. Luiza Ciepielewska-Kaczmarek, Dr. Monika Kowalonek-Janczarek

Redaktionsbeirat Editorial / Advisory Board

Prof. Dr. Jerzy Bańczerowski, Prof. Dr. Anna Cieślicka, Prof. Dr. Józef Darski,

Prof. Dr. Jacek Fisiak, Prof. Dr. Antoni Markunas, Prof. Dr. Kazimiera Myczko,

Prof. Dr. Stanisław Puppel, Prof. Dr. Christoph Schatte, Prof. Dr. Teresa Tomaszkiewicz, Prof. Dr. Weronika Wilczyńska, Prof. Dr. Stephan Wolting, Prof. Dr. Władysław Woźniewicz

Wissenschaftlicher Beirat / Academic Advisory Board

Prof. Dr. Rupprecht S. Baur (Duisburg/Essen), Prof. Dr. Dmitrij Dobrovolskij (Moskau/Wien), Prof. Dr. Franciszek Grucza (Warszawa), Prof. Dr. Wolfgang Herrlitz (Utrecht),

Prof. Dr. Hanna Komorowska (Warszawa), Prof. Dr. Bogdan Kovtyk (Halle),

Prof. Dr. Frank Königs (Marburg/L.), Prof. Dr. Roman Lewicki (Wroclaw),

Prof. Dr. Clare Mar-Molinero (Southhempton), Prof. Dr. Paweł Mecner (Szczecin),

Prof. Dr. Bernd Müller-Jacquier (Bayreuth), Prof. Dr. David S. Singleton (Dublin), Prof. Dr. Marian Szczodrowski (Gdańsk), Prof. Dr. Iwar Werlen (Bern), Prof. Dr. Jerzy Żmudzki (Lublin) 
UNIWERSYTET IM. ADAMA MICKIEWICZA W POZNANIU

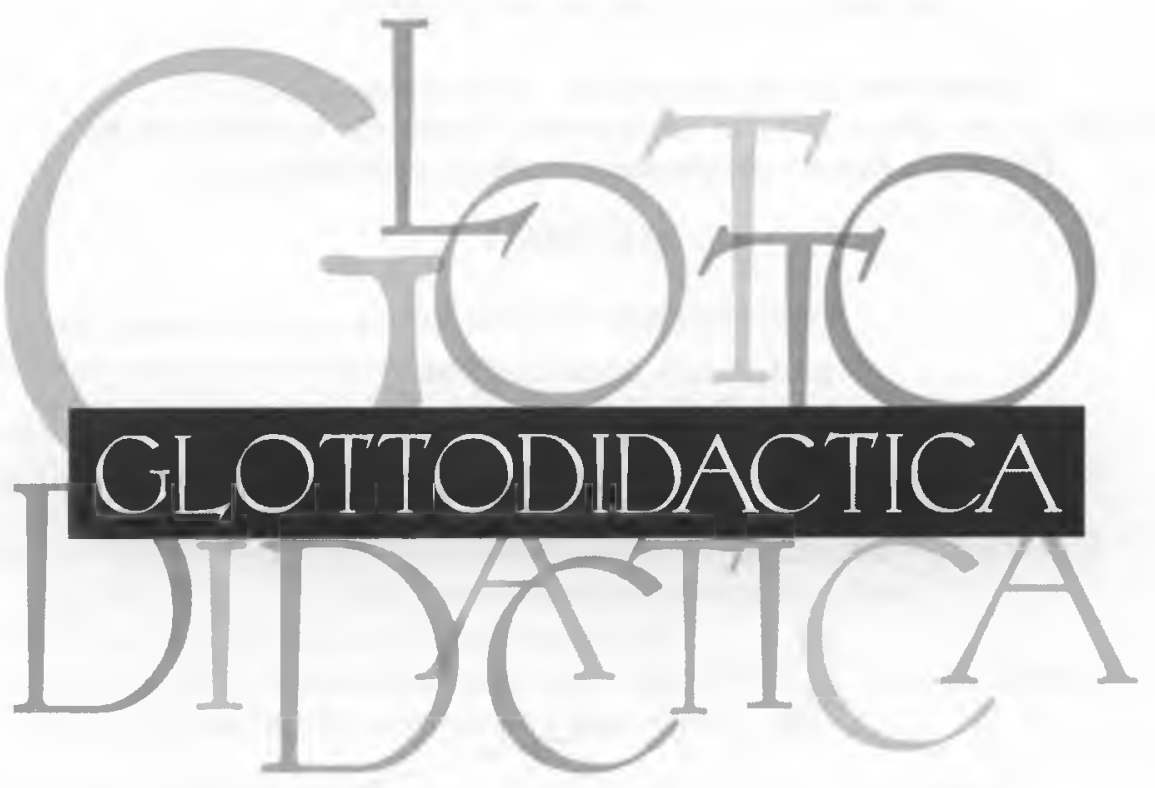

VOLUME XXXV

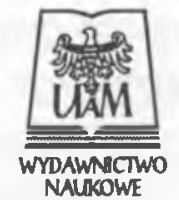

POZNAŃ 2009 


$$
\begin{gathered}
\text { Adres redakcji } \\
\text { Instytut Lingwistyki Stosowanej UAM } \\
\text { ul. } 28 \text { Czerwca } 1956 \text { r. nr } 198 \\
\text { 61-485 Poznań, Poland } \\
\text { tel. }+48618292925 \text {, tel./ fax }+48618292926
\end{gathered}
$$

Editor: Prof. Barbara Skowronek barbaras@amu.edu.pl Assistants to the Editor: Dr Luiza Ciepielewska-Kaczmarek luizac@poczta.fm

Dr Monika Kowalonek-Janczarek monika.kowalonek@wp.pl

$$
\begin{gathered}
\text { Weryfikacja językowa tekstów } \\
\text { prof. dr hab. Anna Cieślicka } \\
\text { dr Britta Stöckmann }
\end{gathered}
$$

Publikacja dofinansowana przez

Instytut Lingwistyki Stosowanej UAM

\section{POZNANI}

(C) Wydawnictwo Naukowe UAM, Poznań 2009

Wydano na podstawie maszynopisu gwarantowan go

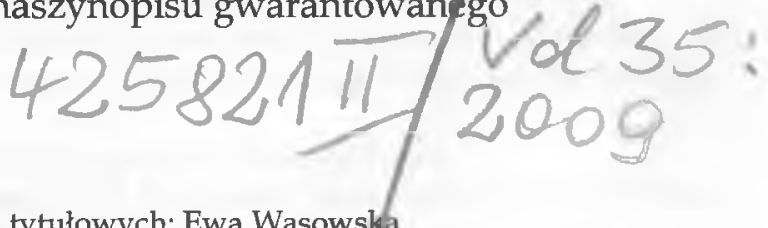

Projekt okladki i stron tytułowych: Ewa Wąsowska

Redaktor prowadzący: Anna Rąbalska

Formatowanie: Eugeniusz Strykowski

\section{ISBN 978-83-232-2083-1 ISSN 0072-4769}

WYDAWNICTWO NAUKOWE UNIWERSYTETU IM. ADAMA MICKIEWICZA

61-734 Poznań, ul. F. Nowowiejskiego 55, tel. 06182939 85, fax 0618293980 e-mail: press@amu.edu.pl www.press.amu.edu.pl

Ark. wyd. 17,00 . Ark. druk. 15,50

ZAKŁAD GRAFICZNY UAM, POZNAŃ, UL. H. WIENIAWSKIEGO 1

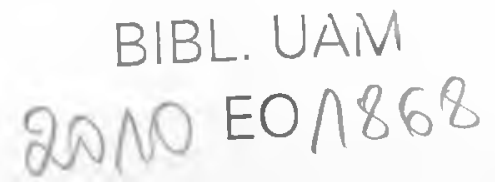




\section{CONTENTS}

\section{ARTICLES}

Basic and Referential Disciplines

Grundlagen- und Referenzwissenschaften

MARIAN SZCZODROWSKI, Wesen, Arten und Wirkungen der fremdsprachlichen Steuerung ...

GRAŻYNA LEWICKA, Zu einigen Problemen des Verstehens in der konstruktivistisch orientier-

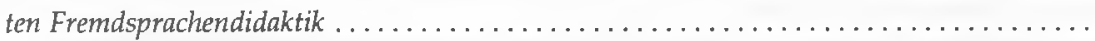

REINHOLD UTRI, Bilinguale Erziehung - neue Herausforderungen und Lösungen aus linguisti-

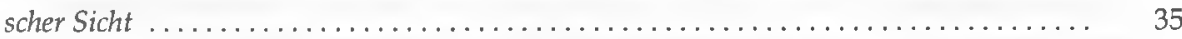

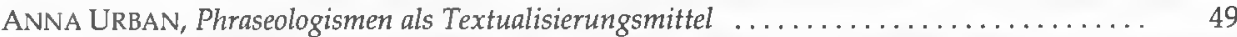

NATASCHA JOURDY, НарратиВная структура художественного текста: свободнокосвенный дискурс как категория лингвистики и нарратологии ................

\section{Methodology in Glottodidactics}

Fremdspracheninethodik

ZOFIA CHLOPEK, The relationship between language learning experience, motivation and some

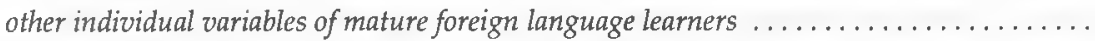

MALGORZATA CZARNECKA, Die Entwicklung interner grammatischer Regeln im gesteuerten

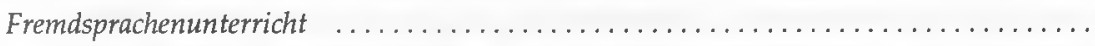

BRIGITA KOSEVSKI PULJIĆ, Der Enwerb der Schreibkompetenz im Deutsch als Fremdsprache -

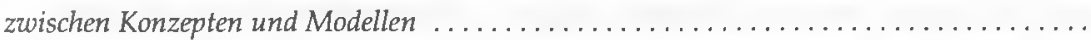

AGNIESZKA MAC, Einige Überlegungen zur Wortbildungslehre im fremdsprachlichen

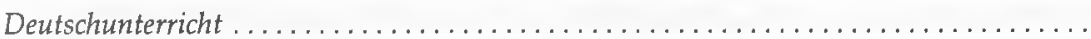

SYlWIA ADAMCZAK-KRYSZTOFOWICZ, ANTJE STORK, Podcasts im glottodidaktischen Medienverbund. Versuch einer vergleichenden Analyse ......................

MARIUSZ WALORCZYK, Optimierung der ZD-Prüfungsvorbereitung in Bezug auf die Aufgabenformen

\section{Language Policy and Intercultural Studies}

Sprachenpolitik und Interkulturelle Studien

MONIKa KOWALONEK-JANCZAREK, Die Nachbarsprache Polnisch aus einer sprachpolitischen und soziolinguistischen Perspektive im Kontext der Mehrsprachigkeit in Europa .........

NINO LOLADZE, Interkulturelles Lernen als Schlüsselqualifikation im Zeitalter der Globalisierung im Kontext des Deutschunterrichts in Georgien 


\section{RESEARCH REPORTS}

DANUTA WIŚNIEWSKA, EFL Teachers' Perception and Practice of Action Research .......... 163

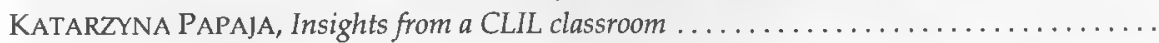

MAGDAlENA KOPER, MAGDALENA DUdZIŃSKA, Europäisierung der Sprachenpolitik im Bereich des Kulturtourismus nach dem EU-Beitritt Polens am Beispiel der Stadt Gdansk ....

\section{BOOK REVIEWS}

BARBARA SKOWRONEK, Marian Szczodrowski: Fremdsprachliche Lehr-Lern-Vorgange im kodematischen Blickfeld. Wydawnictwo Uniwersytetu Gdańskiego, Gdańsk - Sopot 2009,225 S. . . . . . . . . . . . . . . . . . . . . . . . . . . . . . .

JOANNA KIC-DRGAS, Sambor Grucza: Lingwistyka języków specjalistycznych. Wydawnictwo Euro-Edukacja, Warszawa 2008, 256 S.

ANNA PIECZYNSKA-SUlIK, Hans-Jörg Schwenk: Präfigierung im Polnischen und ihre Entsprechung im Deutschen. $\mathrm{Zu}$ Theorie von und lexikographischem Umgang mit Aspekt und Aktionsart. Reihe: Języki - Kultury - Teksty - Wiedza. Band 8, Wydawnictwo EuroEdukacja, Warszawa 2007, $301 \mathrm{~S}$.

BARBARA SKOWRONEK, Magdalena Olpińska: Nauczanie dwujęzyczne w świetle badań $i$ koncepcji glottodydaktycznych. Seria: Języki - kultury - teksty - wiedza. Franciszek Grucza, Jerzy Lukszyn (Hg.). Wydawnictwo Euro-Edukacja, Warszawa 2008, 247 S. . . . . . .

REINHOLD UTRI, Marc Bielefeld: We spe@k Deutsch... aber verstehen nur Bahnhof. Unterwegs im Dschungel unserer Sprache. Wilhelm Heyne Verlag, München, 2008, 286 S. .

GABRIELA GORĄCA, Sambor Grucza: Od lingwistyki tekstu do lingwistyki tekstu specjalistycznego. Seria: Języki - kultury - teksty - wiedza. Franciszek Grucza, Jerzy Lukszyn (Hg.). Wydawnictwo Euro-Edukacja, Warszawa 2007, 218 S.

KATARZYNA KONSEK, Jörg Roche: Handbuch Mediendidaktik. Hueber Verlag, Ismaning $2008,176 \mathrm{~S}$.

ANNA URBAN, Christiane Hümmer: Synonyme bei phraseologischen Einheiten. Eine korpusbasierte Untersuchung. Peter Lang, Frankfurt am Main 2009, 357 S. . . . . . . . .

SYLWIA ADAMCZAK-KRYSZTOFOWICZ, Kazimiera Myczko, Barbara Skowronek, Władyslaw Zabrocki (red.): Perspektywy glottodydaktyki $i$ jezykoznawstwa. Tom jubileuszowy $z$ okazji 70. urodzin Profesora Waldemara Pfeiffera. Wydawnictwo Naukowe UAM, Poznań 2008, 499 S. . . . . . . . . . ..............................

MONIKA KOWALONEK-JANCZAREK, Boris Blahak, Clemens Piber (Hg.): Deutsch als fachbezogene Fremdsprache in Grenzregionen. Ekonóm, Bratislava 2008, 322 S. . . . . . . .

LUIZA CIEPIELEWSKA-KACZMAREK, Sambor Grucza (Hg.): W kręgu teorii i praktyki lingwistycznej. Księga jubileuszowa poświęcona Profesorowi Jerzemu Lukszynowi z okazji 70. rocznicy urodzin. Wydawnictwo Uniwersytetu Warszawskiego, Warszawa 2007, 367 S. . . . . . . . .

AGNIESZKA PAWŁOWSKA, Kultury i języki poznawać - uczyć się - nauczać / Kulturen und Sprachen verstehen - lemen - lehren. Pod redakcją / Herausgegeben von Anna Jaroszewska, Marta Torenc. Uniwersytet Warszawski, Instytut Germanistyki, Warsza-

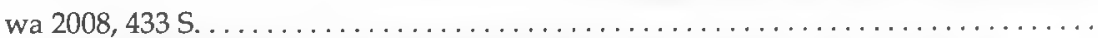

BEATA MikOŁAJCZYK, Konrad Ehlich, Dorothee Heller (Hg.): Die Wissenschaft und ihre Sprachen. 2006. Peter Lang Verlag, Bern / Berlin / Bruselles / Frankfurt a.M. / New York / Oxford / Wien. (= Linguistic Insights. Studies in Language and Communi-

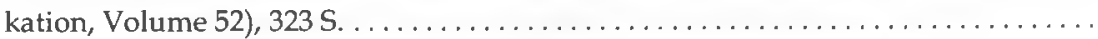




\section{REPORTS}

LUIZA CIEPIELEWSKA-KACZMAREK, Lesen und Leseverstehen in der DaF-Didaktik

MAGDAlena JUREWICZ, PAWEE RYBSZLEGER, Bericht über die Internationale wissenschaftliche Konferenz des Verbandes Polnischer Germanisten (VPG) ..........

AGNIESZKA PAWŁOWSKA, Bericht über die internationale Konferenz Reflexion als Schlüsselphänomen der gegenwärtigen Fremdsprachendidaktik

GRAŻYNA ZENDEROWSKA-KORPUS, Das linguistisch-hochschuldidaktische Kolloquium „Fachsprachenpropädeutik im Germanistikstudium" 



\title{
Zu einigen Problemen des Verstehens in der konstruktivistisch orientierten Fremdsprachendidaktik
}

\section{Understanding in Foreign Language Teaching Process from Constructivist Viewpoint}

\begin{abstract}
The aim of this paper is to justify the assumption that understanding is the essence of learning. From the constructivist viewpoint understanding is a complex mental and social process that involves decoding the symbolic message received from others and then interpreting and assigning a personal meaning to that message. Since personal background largely determines how the message will be understood it is important that the development of the learning environment encourages understanding from multiple perspective. Therefore the learning process should be based on techniques drawn from the constructivist's epistemological assumptions, e.g., construction of intersubjective perspective, situated cognition in authentic life contexts and collaborative learning environment.
\end{abstract}

Keywords: constructivism, constructivist viewpoint of learning, understanding, interpreting, construction of intersubjective perspective, cognition, representation, mental lexicon, learning process, collaborative learning environment.

Verstehen sollte als ein komplexes soziales und kognitives Geschehen zwischen Interaktions- bzw. Kommunikationspartnern begriffen werden (Rusch 1992: 216). Diese These bedarf ein paar weiterer Erklärungen zu den Fragen:

- was hier unter dem sozialen und dem kognitiven Phänomen aufzufassen wäre,

- welche Bedeutung in diesem Zusammenhang die Interaktion und die Kommunikation hätten, 
- welche Art von Verstehen hier gemeint ist,

- welche Konsequenzen diese Auffassung des Verstehens für die Fremdsprachendidaktik hätte.

Um all diesen Problemen Rechnung tragen zu können, möchte ich zunächst kurz auf die systemisch-konstruktivistische Erkenntnistheorie eingehen, die Menschen als autopoietische Systeme betrachtet. Autopoietische Systeme im Unterschied zu allopoietischen (z.B. Rechnern und anderen Maschinen) besitzen autonome Gehirne, die fähig sind, verschiedene kognitive Operationen auszuführen. Zu den wichtigsten gehört die Verarbeitung der von Rezeptoren wahrgenommenen Signale aus der Umgebung. In dieser Weise entstehen Vorstellungen, quasi mentale Repäsentationen der Wirklichkeit. Es sind aber keine mimetischen Abbildungen der Wirklichkeit, sondern subjektive Konstrukte, die durch die bisherige Erfahrung und das aktuelle Weltwissen des Individuums beeinflusst werden (Maturana 1970; Maturana/Varela 1987; Glasersfeld 2003). Auf dieser Grundlage wird es zu einem wichtigen Ansatzpunkt des Konstruktivismus, dass Kommunikation kein Transport von Information sei, weil Bedeutungen sich nicht übermitteln lassen. Das, was menschliche Rezeptoren wahrnehmen können, sind lediglich optische und akustische Signale physikalischer Natur. Erst im Gehirn finden biochemische Prozesse statt und im Laufe der Verarbeitungsprozesse werden den Signalen Bedeutungen zugewiesen. Das meint, die Bedeutungen sind Konstrukte des menschlichen autopoietischen Gehirns. Darüber, was im Gehirn wie verarbeitet wird, entscheiden die sich nach Erfahrung und Wissen des Individuums richtenden Bewertungskriterien:

Je konstruktiver das Gehirn wird, das heißt, je mehr Erfahrung aufgrund rekursiver Hypothesenbildung es besitzt, desto genauer kann es sich an der Welt orientieren. Dies bedeutet, daß das Gehirn aufgrund der internen rekursiven Bewertung der Folgen seines Handelns immer offener für die Umwelt wird, »offener" in dem Sinne, daß es Signalen aus der Umwelt immer schneller und eindeutiger Bedeutungen zuweist (Roth 1996: 364).

Bedeutungen werden entsprechend den im mentalen Lexikon existierenden Konzepten zugewiesen. Der Konzeptualisierungsprozess ist jedem Menschen durch seine Sprache vorgegeben. Das Kind beobachtet die sich wiederholenden Ereignisabläufe in seiner Umgebung und bildet auf dieser Basis senso-motorische Schemata. Mit der Zeit entwickeln sich bei ihm artikulatorische Schemata (selbstverständlich auch in der Interaktion mit seiner Umgebung). Das Kind kommt in ein höheres Entwicklungsstadium, in dem die Objektpermanz nicht mehr nötig ist, weil sie durch die mentale Repräsentation vertreten wird. Das heißt, das Kind beginnt die Artikulationsfolgen als sprachliche Zeichen zu gebrauchen (Piaget 1948). 
Verstehen ist ein individuelles, kognitives Geschehen: Bedeutungen werden den Konzepten im mentalen Lexikon subjektiv zugewiesen. Diese Tatsache hat wichtige Konsequenzen für jede Kommunikationssituation (die sog. Orientierungssituation). Als solche sollte man auch jede didaktische Veranstaltung (nicht nur den schulischen Unterricht, sondern auch Seminare, Vorträge u. dgl. im universitären Bereich) betrachten. Der/die Orientierende kann nie die Gewissheit haben, dass der/die Orientierte den Orientierungswert so aufgefasst hat, wie er/sie es gemeint hatte. Daher sollte das Verstehen laut Rusch (1992) nicht nur als ein kognitives, sondern immer zugleich als ein soziales Geschehen aufgefasst werden. Lediglich in der Interakion und Kommunikation kann man eine Verallgemeinerung (Intersubjektivierung) des Verstehens erlangen.

Wie ich schon früher erwähnt habe, ist jedem Menschen in erster Linie subjektives Verstehen eigen. Lautliche Klänge oder Schrift sind Medien, deren Aufgabe ist, sprachliche Äußerungen nach innen, d. h. ins Gehirn zu übermitteln. Dort finden kognitive Prozesse, die sog. Verstehenshandlungen, statt. Es treten ein: assoziative und inferentielle Verknüpfung von kognitiven Schemata, Querverbindungen zu anderen Wissenskomponenten, Begreifen von Zusammenhängen etc. All dies geschieht auf der Basis von eigener Erfahrung, Weltwissen und dem jeweiligen situativen Kontext. Im Fall des subjektiven Verstehens besteht keine Sicherheit, dass Orientierungserwartungen (objektives Verstehen) erfüllt werden. Daher ist hier der soziale Aspekt wichtig: In Interaktion und Kommunikation wird das Verstehen durch Zustimmung des Orientierenden oder Übereinstimmung mit anderen objektiviert und dadurch verallgemeinert. Die Aufhebung der Unsicherheit subjektiven Verstehens bezeichnet man als Intersubjektivierung (Rusch 1992).

Welche Konsequenzen haben diese erkenntnistheoretischen Grundlagen für die Entwicklung der Verstehensfertigkeit?

Ohne Verstehen ist der Lernvorgang kaum denkbar, es sei denn es geht um einfaches automatisches Auswendiglernen. Verstehen bedingt nämlich weitere kognitive Prozesse (Assoziieren, Inferieren, Behalten, Erinnern u. dgl.), die beim Konstruieren von neuem Wissen und Können unerlässlich sind (Lewicka 2004). Verstehen ist auch eine Voraussetzung für Sprache. Als Begründung dieser These können Forschungen der Entwicklungspsychologen und Psycholinguisten (u. a. Piaget 1948; Bruner 1983; Stern 1985) zum Erstpracherwerb dienen: Kinder erwerben ihre Erstsprache ausschließlich dank der Interaktion mit der Umgebung. Die Forschungsansätze zur kindlichen Kommunikation und Sprachbildung sollten auch für Fremdprachendidaktiker von Belang sein, weil sie interessante Resultate liefern, die eine Quelle von neuen Ideen gerade für das Fremdsprachenlernen sind. Leider muss man feststellen, dass die Fremdsprachendidaktik nach wie vor unter 
dem Einfluss der deskriptiven Linguistik steht. Viele Lehrende bevorzugen den Grammatikunterricht: Die Regeln und der Wortschatz werden auswendig gelernt. Es ist der kürzeste Weg, um grammatisch korrekte Sätze zu bauen. Ein solches Vorgehen hat jedoch gar nichts mit der Entwicklung der Kommunikationfähigkeit zu tun.

Und wie verlaufen die Verstehensprozesse im Unterricht? Viele Lehrende gehen von der (übrigens) ganz falschen Annahme aus: übersetzen zu können heiße zugleich verstanden zu haben. Die Lernenden müssen daher Texte, einfache Sätze ohne Kontext oder sogar lose Wörter in die L1 übersetzen, um zu beweisen, was sie verstanden haben. Eine Wort-für-Wort-Übersetzung ist eine der beliebtesten Techniken in der unterrichtlichen Praxis. Weswegen? Weil sie nicht viel Mühe, gar keine Kreativität seitens der Lehrenden verlangt und den Lernenden zeigt, dass man ohne Auswendiglernen gar nicht auskommen kann. Tatsächlich aber erschwert dieses Verfahren erheblich den Spracherwerb, weil man keinen direkten Bezug zur Wirklichkeit hat, sondern sich ständig zwischen zwei sprachlichen Systemen bewegt: dem L1 und dem einer Fremdsprache.

Der fremdsprachliche Unterricht sollte daher möglichst genau die Wirklichkeit des Landes simulieren. Der situative Kontext schafft gute Bedingungen für die Ausführung von Verstehenshandlungen. In der Interaktion mit der Umgebung, auf Grund allgemeinen Weltwissens und individueller Erfahrung, werden im Gehirn des/der Lernenden kognitive Prozesse aktiviert. Assoziieren und Inferieren unterstützen die Verarbeitung von Information (d.h. Auffassen und Begreifen) und erleichtern dadurch das Verstehen. Die Tatsache, dass die Zuweisung von Bedeutungen durch individuelle Eigenschaften des Menschen geprägt wird, schließt die Überzeugung aus, dass man die Bedeutungen jemandem übermitteln oder sogar „beibringen“ könne.

In der Fremdsprachendidaktik erwähnt man oft den Begriff Semantisierung als einen Unterrichtsteil oder eine Unterrichtsphase, in der neuer Wortschatz, neue Strukturen, neues grammatisches Phänomen etc. eingeführt und erklärt werden. Die meisten Lehrenden betrachten Semantisierung als Vermittlung von bestimmten Informationen in Form eines Vortrags. Niemand macht sich dabei Gedanken, welche Prozesse sich dann in den Gehirnen der Lernenden vollziehen, als wäre es selbstverständlich, dass sie alles genau so wie Diktiergeräte aufnehmen. Eine solche Auffassung der Semantisierung finde ich nicht richtig (Lewicka 2007a, 2007b, 2007c), denn es werden hier die grundlegenden Bedingungen für die Ausbildung der Verstehensfertigkeit nicht erfüllt.

Am Anfang meines Beitrags habe ich die These angeführt, dass Verstehen ein komplexes soziales und kognitives Geschehen zwischen Interaktionsbzw. Kommunikationspartnern ist. Die Verallgemeinerung des Verstehens 
(sog. objektives Verstehen) kann nur in einer Kommunikaktionssituation zustande kommen und nicht im Frontalunterricht, wenn jemand „etwas" vorträgt oder sogar vorliest und die anderen müssen den Erwägungen nur folgen, ohne eine Möglichkeit zu haben, ihr subjektives Verstehen eventuellen Korrekturen zu unterziehen. Zudem bekommt der/die Orientierende (d.h. der/die Vortragende) auch keine Rückmeldung, wie die Verstehensprozesse bei den Orientierten (d. h. bei den Lernenden) verlaufen. Dieses Problem gewinnt umso mehr an Bedeutung, wenn die zu verstehenden Informationen in einer oder über eine Fremdsprache geäußert werden. In dem Fall ist die Intersubjektivierung besonders wichtig: Die Bewertungskriterien zur Selektion von Kognitionen sind historisch und kulturell bedingt und so stark internalisiert, dass die Lernenden (hier: die Orientierten) eine 'fremde' Wirklichkeit mit ihren eigenen Kriterien auffassen und begreifen. Das führt offensichtlich zu Missverständnissen.

Die Tatsache, daß ein und derselbe Vorgang, ein und dasselbe Resultat Verstehen und Miß-Verstehen sein kann, zeigt uns das Gewicht der autonomen, nichtsprachlichen Faktoren in diesem Geschehen (Hörmann 1994: 500).

Wie sollte jedoch ein solches Geschehen aus fremdsprachlicher Sicht beurteilt werden? Auf wessen Seite liegt hier die Schuld? Wer hat diesen Vorfall verursacht? Der/die Orientierende oder der/die Orientierte? Selbstverständlich hat hier der/die Orientierende einen Fehler begangen, denn es gehörte zu seinen/ihren Aufgaben, das Verstehen in Interaktion und Kommunikation zu intersubjektivieren. Das meint, die Lernenden (seien es Schüler oder Studenten) sollten die Möglichkeit bekommen, divergentes Denken zu entwickeln: Meinungen zu äußern, offene Fragen zu stellen sowie zu beantworten. Auf diesem Weg wären sie imstande, ihr Wissen zu konstruieren und nicht nur Notizen niederzuschreiben (ihrer Meinung nach, möglichst 'genau', was sie gehört haben), um dann alles auswendig zu lernen. Zu bedauern ist die Tatsache, dass dies nicht nur die schulische, sondern auch die universitäre Gewohnheit ist. Zeitmangel ist, nach meiner Ansicht, keine Ausrede. Lernen ist doch ein langwieriger Prozess und wir alle, die mit Didaktik zu tun haben, sind eigentlich dazu da, für die Schüler/Studenten optimale Arbeitsbedingungen zu schaffen. Lernen muss man selbst, es ist nicht möglich, Wissen zu transportieren, wie es im Falle von Vorträgen (eine längere Zeit zuhören und zugleich möglichst viel notieren) unterstellt wird.

Beim Fremdsprachenlernen dominiert auch die Überzeugung, dass man alles Wort-für-Wort verstehen sollte. Diese Vorgehensweise ist analytischen Charakters und ähnelt der Bottom-up-Verarbeitung, wobei man schrittweise zur Bedeutung einer Äußerung gelangt, indem man mit der artikulatorischen und syntaktischen Analyse anfängt. Es kommt vor, dass die Lehren- 
den die Verstehensprozesse durch die Übersetzung in die L1 beschleunigen und erleichtern wollen. Es ist aber eine ganz falsche Auffassung des Verstehens. Die Konfrontation mit der L1 in der ersten Etappe des Fremdspracherwerbs schadet der weiteren Entwicklung der Sprechfertigkeit, weil sie die Denkprozesse bei der Planung einer Äußerung verlangsamt und verschiedenartige Interferenzfehler verursacht. Der Lerner, der noch zu wenig Erfahrung im Umgang mit der zu erlernenden Sprache hat, versucht Konzepten fremdsprachliche Wortformen zu geben (als Konzept bezeichnet Kintsch (1974) eine mentale nicht sprachgebundene Einheit im mentalen Lexikon). Eine andere Schwierigkeit bereitet die Entscheidung, welchem der Konzepte eine neue Bedeutung zuzuweisen wäre, wenn man nur eine L1-Übersetzung bekommen hat, ohne jeglichen Bezug auf gewisse Gegenstände und Sachverhalte.

Um näher erläutern zu können, welche Zusammenhänge zwischen Verstehen und Sprechen bestehen, gehe ich nochmals auf die Probleme des Verstehens ein. Am Beispiel des mentalen Modells von Johnson-Laird (1989) werde ich eine Theorie präsentieren, die meines Erachtens, die Entwicklung des Verstehens in der Fremdsprachendidaktik optimieren könnte, weil sie besser den kommunikativen Aspekt dieses Prozesses berücksichtigt und dadurch zur Förderung der Sprechfertigkeit beizutragen vermag. Als Grundlage der Theorie wurde der Top-down-Ansatz angenommen: Beim Verstehen geht es darum, eine mentale Repräsention aufzubauen, die möglichst gut der Struktur des betreffenden Sachverhalts entspricht. Im Gegenteil zum Bottom-up-Ansatz werden hier zu Beginn extralinguistische Elemente verarbeitet, die bestimmte Schemata aktivieren und die Prozesse des Antizipierens und Inferierens verursachen. Das Interessanteste in der Theorie ist, dass sich hier die Top-down- und die Bottom-up-Prozesse verflechten, was die Verstehenshandlungen positiv beinflussen kann.

Dieses Modell könnte in der Fremdsprachendidaktik Anwedung finden, aber lediglich unter der Bedingung, dass man einen richtigen Kontext organisiert, der die außersprachliche Wirklichkeit des Zielsprachenlandes simuliert. Der direkte Bezug auf die Wirklichkeit erleichtert das Verstehen, man muss sich nicht nur auf die Sprache konzentrieren, Gesten, Mimik und andere extra- sowie paralinguistische Elemente der Kommunikation sind gute Verstehenshilfen. Dabei werden die Denkprozesse schon von Anfang an auf den Gebrauch einer fremden Sprache eingestellt (umgangssprachlich wird das als Denken in einer Fremdsprache bezeichnet). Eine gute Voraussetzung dafür und zugleich für die Sprachproduktion ist die nach dem mentalen Modell entwickelte Verstehensfertigkeit.

Die Sprachproduktion, aufgefasst im Sinne der kommunikativen Kompetenz von Hymes (1971) als Sich-sprachlich-verhalten-können, umfasst vier 
Ebenen (pragmatische, semantische, morpho-syntaktische und motorische) und verläuft inkrementell. Inkrementelle Verarbeitung beruht darauf, dass die unteren Ebenen aktiv werden können, bevor die Verarbeitung auf der höheren Ebene abgeschlossen ist (die Planung einer Äußerung auf der pragmatischen Ebene ist noch nicht zu Ende, wenn die semantische aber schon anfängt). Die von Levelt (1999) konzipierte autonome Theorie der Sprachproduktion fasst die vier Ebenen (pragmatische, semantische, morphosyntaktische und motorische) in drei Verarbeitungsstufen zusammen: Den Konzeptualisator für den vorsprachlichen Inhalt (pragmatische und semantische Ebenen), den Formulator für die Versprachlichung des vorsprachlichen Inhalts (morpho-syntaktische Ebene) und den Artikulator (artikulatorische Ebene). Die drei Verarbeitungsstufen wurden zwei Systemen zugeordnet: dem semantisch-syntaktischen und dem phonologisch-phonetischen.

Bei der Analyse des Modells der Sprachproduktion kann man erklären, in welchem Moment Lerner, die durchs Übersetzen in die L1 eine Fremdsprache erlernen, auf die Schwierigkeiten stoßen, wenn sie sich in einer Fremdsprache äußern wollen. Sie planen die Strategie für ihre Äußerung in L1 und, wenn es zur Verprachlichung kommt, suchen sie nach Strukturen, die genau denen ihrer L1 entsprechen. Um diese Fehler zu vermeiden, sollte man das deklarative Wissen (das sind Repräsentationen im mentalen Lexikon) operationalisieren, das heißt in das prozedurale Wissen überführen. Das prozedurale Wissen umfasst kognitive Prozesse, die u. a. für sprachliche Handlungen zuständig sind (Anderson 1996). Daher sollte der Fremdsprachenunterricht in Form von Episoden geplant werden, die an das episodiche Gedächtnis addressiert werden (Lewicki/Lewicka 1999). Auf diese Weise entstehen im Gedächtnis globale Muster von Ereignissen und Zuständen in Form von Repräsentationen (Beaugrande/Dressler 1981), zum Beispiel Frames (globale Muster, die Alltagswissen über bestimmtes zentrales Konzept umfassen) oder Schemata (globale Muster von Ereignissen und Zuständen in geordneten Abfolgen).

Abschließend seien noch einmal die wichtigsten Punkte genannt, die ich in meinem Beitrag betrachtet habe:

- Wissen wird im menschlichen Gehirn konstruiert.

- Grundlage für das Konstruieren von Wissen sind eigene Erfahrungen und bisheriges Weltwissen.

- Verstehen ist die wichtigste Bedingung für erfolgreiches Lernen.

- Verstanden zu haben meint: deklaratives Wissen (Repräsentationen) in prozedurales Wissen (kognitive Prozesse) umsetzen zu können.

- Diese Umsetzung ist nur dann möglich, wenn den Konzepten im mentalen Lexikon neue Bedeutungen zugewiesen werden.

- Für das Fremdsprachenlernen ist das prozedurale Wissen von besonderer Bedeutung. 
- Weder deklaratives noch auch prozedurales Wissen lassen sich übermitteln.

- Auswendiglernen schließt das Verstehen aus, weil es keine kognitive Leistung erbringt.

- Übersetzen in die L1 beeinträchtigt das Fremdsprachenlernen, weil die Lerner dabei keinen direkten Bezug zur außersprachlichen Wirklichkeit haben.

- Verstehen ist ein subjektiver psychischer Prozess, deswegen muss es intersubjektiviert werden.

- Diese Intersubjektivierung (Verallgemeinerung des Verstehens) findet in Interaktion und Kommunikation statt.

- Die Verallgemeinerung des Verstehens führt zum 'objektiven Verstehen', das den Orientierungserwartungen entsprechen sollte.

- Da Sprechen ein soziales Verhalten ist, sollte der Fremdsprachenunterricht in Form von Episoden geplant werden.

- Auf diese Weise entstehen im episodischen Gedächtnis der Lerner globale Muster von Ereignissen und Zuständen.

- Die Lerner erwerben mithin ein richtiges sprachliches Verhalten, das viel wertvoller ist als das grammatisch korrekte Formulieren von Sätzen.

\section{LITERATURVERZEICHNIS}

Anderson, J. R., 1996. Kognitive Psychologie. Heidelberg: Spektrum.

Beaugrande, R.-A. de/Dressler, W.U., 1981. Einführung in die Textlinguistik. Tübingen: Niemeyer.

Bruner, J., 1983. Child's talk: Learning to use language. New York: Niemeyer.

Johnson-Laird, P.N., 1989. Mental model. In: M.I. Posner (Hrsg.): Foundations of cognitive science. Cambridge, MA.: MIT Press, 467-499.

Glasersfeld, E. von, 2003. Konstruktion der Wirklichkeit und des Begriffs der Objektivität. In: H. Gumin/H. Meier (Hrsg.): Einführung in den Konstruktivismus. München: Piper, 9-49.

Hörmann, H., 1994. Meinen und Verstehen. Grundzüge einer psychologischen Semantik. Frankfurt am Main: Suhrkamp.

Hymes, D., 1971. On Communicative Competence. Philadelphia: University of Pennsylvania.

Kintsch, W., 1974. The representation of meaning in memory. Hillsdale: Erlbaum.

Levelt, W.J.M., 1999. Producing spoken language: a blueprint of the speaker. In: C.M. Brown /P. Hagoort (Hrsg.), The neurocognition of language. Oxford: Oxford University Press, 83-122.

Lewicka, G., 2004. Zu einigen Problemen der Glottodidaktik aus der Perspektive der konstruktivistisch-systemischen Kognitionstheorie. In: Kwartalnik Neofilologiczny LI/3, 257-262.

Lewicka, G., 2007a. Glottodydaktyczne aspekty akwizycji jezyka drugiego a konstruktywistyczna teoria uczenia się. Wroclaw: ATUT.

Lewicka, G., 2007b. Zum Konzept des diskursiven Zweitspracherwerbs. In: B. Stein (Hrsg.), Wege zu anderen Sprachen und Kulturen. Hamburg: Dr. Kovač, 151-158. 
Lewicka, G., 2007c. Prozeduren als Diskurshandlungen im glottodidaktischen Prozess. In: Glottodidactica XXXIII, 21-28.

Lewicki, R. /Lewicka, G., 1999. Didaktisch-methodische Konzeption zum Lehrwerk. In: $\mathrm{Na}$ und? Poradnik dla nauczyciela. Testy. Materiaty do kopiowania. Warszawa: PWN, 7-18.

Maturana, H., 1970. Biology of cognition. Urbana. Illinois: University of Illinois.

Maturana, H./Varela F.J., 1987. Der Baum der Erkenntnis. Bern, München, Wien: Scherz.

Piaget, J., 1948. Le langage et la pensée chaz l'enfant. Neuchâtel: Delachaux u. Niestlé.

Roth, G., 1996. Die Konstruktion von Bedeutung im Gehirn. In: S.J. Schmidt (Hrsg.) Gedächtnis: Probleme und Perspektiven der interdisziplinären Gedächtnisforschung. Frankfurt am Main: Suhrkamp, 360-370.

Rusch, G., 1992. Auffassen, Begreifen, Verstehen. In: S.J. Schmidt (Hrsg.): Kognition und Gesellschaft. Der Diskurs des Radikalen Konstruktivismus 2. Frankfurt am Main: Suhrkamp, 214-256.

Stern, D. N.,1985. The interpersonal world of the infant. New York: Basic Books. 
